\title{
Crescimento linear das crianças brasileiras: reflexões no contexto da equidade social
}

\author{
Linear growth of Brazilian children: Reflections \\ in the context of social equity
}

R E S U M O

O artigo teve por objetivo refletir a respeito do crescimento linear das crianças no contexto da equidade social e sistematizou, primeiramente, os resultados das pesquisas que analisaram fatores associados ao declínio do deficit de estatura das crianças menores de cinco anos no Brasil. Posteriormente, os resultados foram contextualizados do ponto de vista da equidade social. Os nove artigos revisados mostraram variação temporal positiva do deficit de estatura, com valores nacionais que oscilaram de 37,1 a 7,1\%; na região Nordeste a variação foi de 33,9 a 5,9\%. Quatro razões principais explicaram o declínio do deficit de estatura: 1) aumento do poder aquisitivo/renda familiar nos lares de baixo poder aquisitivo; 2) melhoria da escolaridade materna; 3) expansão da rede pública de saneamento básico, e 4) expansão dos cuidados básicos de saúde. Porém, destacam-se disparidades que marcam a persistência das iniquidades sociais relacionadas ao deficit de estatura no País, as quais prejudicam populações marginalizadas. Enfoca-se a necessidade de refletir a baixa estatura na perspectiva da influência mediada pela situação da segurança alimentar, do estado nutricional de micronutrientes e dos modos de vida contemporâneos. Na perspectiva das políticas públicas, ressalta-se a importância do trabalho intersetorial. Em conclusão, sugere-se que apesar do declínio do deficit de estatura no Brasil, como reflexo do impacto das políticas públicas, ainda persistem iniquidades com vulnerabilidade marcada em grupos socioculturais desfavorecidos. Assim, o enfrentamento desta conjuntura torna imprescindível o direcionamento das pesquisas e das ações para esses segmentos populacionais.

Palavras-chave: Desigualdades em saúde. Estatura. Iniquidade social. Inquéritos nutricionais. Políticas públicas.

The aim of this study was to assess the linear growth of children in the context of social equity. At first, the study systematized the results of research that examined factors associated with the decline of stunting among children younger than five years of age in Brazil. Then, such results were contextualized from the point of view of social equity. The nine articles reviewed showed a positive temporal variation of stunting, with national values ranging from 37.1 to $7.1 \%$; in Northeastern Brazil, this variation ranged from 33.9 to $5.9 \%$. Four main

\footnotetext{
1 Universidade Estadual da Paraíba, Departamento de Enfermagem, Programa de Pós-Graduação em Saúde Pública. Av. das Baraúnas, 351, Campus Universitário, Bodocongó, 58109-753, Campina Grande, PB, Brasil. E-mail: <dixisfigueroa@gmail.com>.
} 
reasons explained the decline of stunting: 1) increased purchasing power/family income among low-income families; 2) improved maternal education; 3) expansion of public sanitation, and 4) expansion of primary health care services. However, disparities that mark the persistence of social inequalities related to stunting in the country should be highlighted, which affect marginalized populations. There is a need to reflect on stunting from the perspective of influence mediated by food security, nutritional micronutrient status, and contemporary lifestyles. From the perspective of public policies, the results emphasize the importance of intersectoral work. In conclusion, despite the decline of stunting in Brazil as a result of the impact of public policies, there are still inequities with marked vulnerability in disadvantaged sociocultural groups. Thus, the targeting of research and actions for these population segments is imperative.

Keywords: Health inequalities. Body height. Social inequity. Nutrition surveys. Public policies.

\section{N T R O D U Ç Ã O}

O crescimento linear das crianças, além de refletir as condições de vida pregressa, constitui um dos indicadores holísticos da saúde e do bem-estar da população. A sua relevância vai além do significado clínico ou epidemiológico ${ }^{1,2}$. O padrão de crescimento infantil é uma expressão do desenvolvimento humano, das desigualdades nas condições de vida de uma população e um forte preditor do futuro capital humano, do progresso social e da saúde das gerações futuras ${ }^{1,3,4}$.

Apesar das reduções significativas nos países em desenvolvimento, a desnutrição na infância, diagnosticada a partir do retardo do crescimento, continua sendo o principal problema de saúde pública em muitos locais ${ }^{5}$. Reduzir à metade a prevalência de deficit antropométricos em crianças menores de cinco anos constitui uma das metas de desenvolvimento do milênio assumidas em 2000 pelas Nações Unidas ${ }^{6}$. Nesse sentido, desde 2003 o governo brasileiro tem priorizado a eliminação da pobreza e da fome ${ }^{7}$ através de políticas públicas ${ }^{8}$; o impacto positivo das mesmas pode ser analisado por meio do estudo da evolução temporal da distribuição social do deficit de estatura ${ }^{3,9}$. A proeminência dessa constatação importa não só ao Brasil, pois a experiência brasileira pode ser significativa para muitos outros países.

Conhecer as iniquidades socioeconômicas em saúde e nutrição é essencial para conseguir os Objetivos de Desenvolvimento do Milênio. É possível observar, em termos de desnutrição, enormes iniquidades tanto entre diferentes países quanto dentro deles ${ }^{10}$. Em relação ao deficit de estatura, estudo que analisou informações de 47 países de rendas baixa e média mostrou pronunciadas iniquidades socioeconômicas, particularmente nos países da América Latina e no Caribe. Entre os 47 países, o Brasil ocupou o quinto lugar no que diz respeito às injustiças socioeconômicas ${ }^{11}$.

Dessa forma, o presente trabalho tem por objetivo refletir a respeito do crescimento linear no contexto da equidade social. Para tanto, tem-se como base os resultados dos estudos sobre fatores associados ao declínio do deficit de estatura nas crianças brasileiras menores de cinco anos.

\section{MÉ TOD OS}

Este artigo baseia-se nos resultados das pesquisas que analisaram fatores associados ao declínio do deficit de estatura no Brasil. Foram consultadas as bases de dados bibliográficos Scientific Electronic Library Online (SciELO), Literatura Latino - Americana e do Caribe em Ciências da Saúde (Lilacs) e National Library of Medicine (PubMed), Estados Unidos. A busca considerou artigos publicados de 2006 - ano da publicação das novas curvas de crescimento da Organização Mundial da Saúde (OMS) ${ }^{12}$-, a 2015 (8 de fevereiro) nos idiomas inglês, português e espanhol.

Dois conjuntos de intersecção de termos de busca bibliográfica, um referente à nutrição e o outro às desigualdades em saúde, foram com- 
binados. No primeiro caso, os termos utilizados foram: "estado nutricional" ("nutritional status"), "antropometria" ("anthropometry"), "crescimento" ("growth"), "desnutrição" ("malnutrition") e "inquéritos nutricionais" ("nutrition surveys"); já no segundo, foram: "desigualdades em saúde" ("health inequalities"), fatores socioeconômicos ("socioeconomic factors"). Cada termo foi cruzado individualmente com outro, de forma a garantir a inclusão de todos os artigos relacionados ao tema. No caso do PubMed, além dos descritores anteriores, o termo "Brasil" ("Brazil") também foi incluído na busca. Foram analisadas, ainda, as listas de referências dos artigos a fim de identificar outros estudos relevantes, sem levar em consideração, contudo, a data da publicação do artigo nesses casos.

Foram adotados como critérios de inclusão: trabalhos com abordagem na variação temporal; estudos baseados em inquéritos de base populacional representativos do Brasil ou de cidades, regiões ou estados brasileiros; inclusão de crianças menores de cinco anos como população de estudo; e a utilização do deficit de estatura como desfecho de análise. Inicialmente, foram observados o título e o resumo dos estudos, sendo rejeitados aqueles que não atendiam aos critérios de inclusão. Nos casos de incerteza, o texto completo foi consultado para confirmar a elegibilidade do estudo, baseando-se na sua adequação à abordagem proposta.

Os artigos selecionados foram caracterizados segundo local de estudo, inquéritos analisados, tamanho amostral, resultados sobre a variação temporal, resultados sobre fatores associados à evolução do deficit de estatura e conclusões/considerações. Durante essa etapa, estudos que não centravam a atenção nas crianças menores de cinco anos foram identificados e excluídos. Assim, através da análise dos artigos, a presente pesquisa sistematiza, primeiramente, os resultados obtidos para, em seguida, contextualizá-los do ponto de vista da equidade social.

\section{Fatores associados ao declínio do deficit de estatura nas crianças brasileiras menores de cinco anos: sistematização dos resultados}

Na estratégia de busca foram identificados 222 documentos, não duplicados ou triplicados nas bases de dados, dos quais 8 artigos atenderam aos critérios de elegibilidade. Desses, 2 foram considerados em conjunto ${ }^{1}$, uma vez verificada a utilização dos mesmos dados em ambos, diferenciando-se, apenas, nos métodos de análise; além disso, outro artigo foi excluído por não atender ao critério da faixa etária considerada. Dessa forma, 6 artigos foram considerados para a sistematização, sendo que a lista de referências dos mesmos possibilitou a seleção de mais dois estudos.

Do total de artigos ${ }^{1,3,4,13-18}$ (Quadro 1), quatro $3,4,14,16$ referiram-se ao Brasil como um todo. Em conjunto, esses estudos basearam-se nos resultados de quatro inquéritos nacionais: o Estudo Nacional de Despesa Familiar (ENDEF)/1974-1975, a Pesquisa Nacional sobre Saúde e Nutrição (PNSN)/1989, a Pesquisa Nacional de Demografia e Saúde (PNDS)/1996 e a PNDS/2006-2007. Nos outros artigos, os pesquisadores analisaram a situação da região Nordeste ${ }^{13}$ como um todo (baseado em resultados de inquéritos nacionais); dos estados de Pernambuco ${ }^{1}$ (baseado em inquéritos estaduais), São Paulo ${ }^{15}$ (considerando o período de 1974-1975 a 1984-1985 e 1995-1996, baseando-se em dados desagregados do ENDEF/ 1974-1975 e nas pesquisas estaduais de saúde e nutrição de 1984-1985 e 1995-1996) e de Alagoas (SE) ${ }^{18}$ (baseado em inquéritos estaduais); e da cidade de Pelotas (RS) ${ }^{17}$ (baseado nas coortes de Pelotas de 1982, 1993 e 2004).

Observou-se, em todas as análises, variação temporal positiva, com valores nacionais de deficit de estatura oscilando de 37,1 a 7,1\%3,16. As variações para o Nordeste ${ }^{13}$, Pernambuco', São Paulo $^{15}$ e Alagoas ${ }^{18}$ foram, respectivamente, de 33,9 a $5,9 \% ; 12,1$ a $6,4 \% ; 19,5$ a $2,4 \%$ e 22,5 a $11,4 \%$. Na cidade de Pelotas (RS), a maior 
Quadro 1. Caracterização geral dos estudos sobre fatores associados ao declínio do deficit de estatura em crianças menores de cinco anos no Brasil.

\begin{tabular}{|c|c|c|c|}
\hline Fonte & Local de estudo & Inquéritos analisados & Tamanho amostral \\
\hline Figueiroa et al. ${ }^{*}$ & Pernambuco & $\begin{array}{l}\text { IIPESN-PE/1997 } \\
\text { IIIPESN-PE/2006 } \\
\text { IDANT-PE/2006 }\end{array}$ & 1611 \\
\hline Monteiro et al. ${ }^{3}$ & Brasil & $\begin{array}{l}\text { ENDEF/1974-1975 } \\
\text { PNSN/1989 } \\
\text { PNDS/1996, 2006-2007 }\end{array}$ & $\begin{array}{ll}\text { 1974: } 34409 \\
\text { 1989: } 7374 \\
\text { 1996: } 4149 \\
\text { 2006: } 4414\end{array}$ \\
\hline de Onis et al. ${ }^{4}$ & $\begin{array}{l}\text { Países em desenvolvimento, entre os } \\
\text { quais o Brasil }\end{array}$ & $\begin{array}{l}\text { ENDEF/1974-1975 } \\
\text { PNSN/1989 } \\
\text { PNDS/1996 }\end{array}$ & - \\
\hline Lima et al. ${ }^{13}$ & Nordeste & PNDS/1986, 1996, 2006-2007 & $\begin{array}{lr}\text { 1986: } & 1177 \\
\text { 1996: } & 1872 \\
\text { 2006: } & 870\end{array}$ \\
\hline Monteiro et al. ${ }^{14}$ & Brasil & PNDS/1996, 2006-2007 & $\begin{array}{ll}\text { 1996: } & 4132 \\
\text { 2006: } & 4034\end{array}$ \\
\hline $\begin{array}{l}\text { Monteiro \& } \\
\text { Conde et al. }{ }^{15}\end{array}$ & São Paulo & $\begin{array}{l}\text { ENDEF/1974-1975 } \\
\text { IPSNSP/1984-1985 } \\
\text { IIPSNSP/1995-1996 }\end{array}$ & $\begin{array}{ll}\text { 1974: } & 1008 \\
\text { 1984: } & 1016 \\
\text { 1995: } & 1280\end{array}$ \\
\hline Monteiro et al. ${ }^{16}$ & Brasil & $\begin{array}{l}\text { ENDEF/1974-1975 } \\
\text { PNSN/1989 } \\
\text { PNDS/1996, 2006-2007 }\end{array}$ & $\begin{array}{l}\text { 1974: } 34409 \\
\text { 1989: } 7374 \\
\text { 1996: } 4149 \\
\text { 2006: } 4414\end{array}$ \\
\hline Matijasevich et al. ${ }^{17}$ & Pelotas (RS) & $\begin{array}{l}\text { Coorte de Pelotas (RS) de 1982, } 1993 \text { e } \\
2004\end{array}$ & $\begin{array}{ll}\text { 1982: } & 5914 \\
\text { 1993: } & 5249 \\
\text { 2004: } & 4231\end{array}$ \\
\hline Ferreira et al. ${ }^{18}$ & Alagoas (SE) & Inquéritos estaduais de 1992 e 2005 & $\begin{array}{ll}\text { 1992: } & 1228 \\
\text { 2005: } & 1384\end{array}$ \\
\hline
\end{tabular}

Nota: *Refere-se aos artigos: 1) Figueiroa JN, Alves JGB, Lira PIC, Batista Filho M. Evolução intergeracional da estatura no Estado de Pernambuco, Brasil, entre 1945 e 2006. 2 - aspectos analíticos. Cad Saúde Pública. 2012; 28(8):1468-78; e 2) Figueiroa JN, Alves JGB, Lira PIC, Batista Filho M. Evolução intergeracional da estatura no Estado de Pernambuco, Brasil, entre 1945 e 2006.1 - aspectos descritivos. Cad Saúde Pública. 2012; 28(7):1285-96.

PESN-PE: Pesquisa Estadual de Saúde e Nutrição: Saúde, Nutrição, Alimentação, Condições Socioeconômicas e Atenção à Saúde no Estado de Pernambuco; DANT-PE: Inquérito Estadual sobre Doenças Crônicas e Agravos Não Transmissíveis: Prevalência e Fatores de Risco; PNDS: Pesquisa Nacional de Demografia e Saúde; ENDEF: Estudo Nacional de Despesa Familiar; PNSN: Pesquisa Nacional de Saúde e Nutrição; PSNSP: Pesquisa de Saúde e Nutrição das crianças de São Paulo.

variação esteve entre as crianças de dois anos de idade, de 13,9 a 5,0\% ${ }^{17}$. Modelos estatísticos identificaram quatro razões principais para esses declínios: 1) aumento do poder aquisitivo/renda familiar nos lares de baixo poder aquisitivo 1,3,13-17; 2) melhoria da escolaridade materna',13-16; 3) expansão da rede pública de saneamento básico 13-16,18, e 4) expansão dos cuidados básicos de saúde ${ }^{13-16,18}$ (Quadro 2).

Como conclusões/considerações (Quadro 3) convergentes aos resultados, os principais aspectos tratados pelos autores referem-se à necessidade de continuar os investimentos direcionados à melhoria das condições socioeconômicas/poder aquisitivo $0^{1,3,13-16}$, da assistência à saúde ${ }^{13-16}$, da educação ou escolaridade mater$n a^{13-16}$ e do saneamento ${ }^{13,14,16}$. Cabe ressaltar que, apesar de em alguns estudos os pesquisadores terem usado informações dos mesmos inquéritos, os métodos de análise foram diferentes, com capacidade de responder adequadamente às indagações propostas e sem, necessariamente, ter que conduzir a resultados similares. 
Quadro 2. Resultados dos estudos sobre fatores associados ao declínio do deficit de estatura em crianças menores de cinco anos no Brasil.

\begin{tabular}{|c|c|c|c|}
\hline Fonte & Local de estudo & Variação temporal (\%) & $\begin{array}{c}\text { Fatores associados à evolução do } \\
\text { déficit de estatura }\end{array}$ \\
\hline Figueiroa et al..$^{*}$ & Pernambuco & $12,1-6,4$ & $\begin{array}{l}\text { Sexo, escolaridade da mãe, poder aquisitivo fa- } \\
\text { miliar, local de residência, peso ao nascer, reali- } \\
\text { zação de pré-natal, ocorrência de diarreia pré- } \\
\text { via. }\end{array}$ \\
\hline Monteiro et al. ${ }^{3}$ & Brasil & $37,1-7,1$ & Poder aquisitivo familiar. \\
\hline de Onis et al. ${ }^{4}$ & $\begin{array}{l}\text { Países em desenvolvimento, } \\
\text { entre os quais o Brasil }\end{array}$ & $\begin{array}{l}32,0-10,5 \\
\text { Tendência descendente (variação } \\
\text { anual de }-1,02 \text { pontos percen- } \\
\text { tuais) }\end{array}$ & - \\
\hline Lima et al. ${ }^{13}$ & Nordeste & $\begin{array}{l}1986-1996: 33,9-22,2 \\
1996-2006: 22,2-5,9\end{array}$ & $\begin{array}{l}\text { Escolaridade da mãe, poder aquisitivo familiar, } \\
\text { saneamento básico, assistência à saúde, ante- } \\
\text { cedentes reprodutivos. }\end{array}$ \\
\hline Monteiro et al. ${ }^{14}$ & Brasil & $13,5-6,8$ & $\begin{array}{l}\text { Escolaridade da mãe, poder aquisitivo familiar, } \\
\text { saneamento básico, assistência à saúde. }\end{array}$ \\
\hline $\begin{array}{l}\text { Monteiro \& Conde } \\
\text { et al. }{ }^{15}\end{array}$ & São Paulo & $19,5-2,4$ & $\begin{array}{l}\text { Determinantes distais (escolaridade da mãe, po- } \\
\text { der aquisitivo familiar) e intermediários (sanea- } \\
\text { mento básico, assistência à saúde, anteceden- } \\
\text { tes reprodutivos). }\end{array}$ \\
\hline Monteiro et al. ${ }^{16}$ & Brasil & $\begin{array}{l}\text { 1974-1989: } 37,1-19,9 \\
\text { 1989-1996: } 19,9-13,5 \\
\text { 1996-2006: } 13,5-7,1 \\
\text { Quinto mais pobre: } 59,0-11,2 \\
\text { Quinto mais rico: } 12,1-3,3\end{array}$ & $\begin{array}{l}\text { Desigualdade socioeconômica (maior declínio } \\
\text { entre os mais pobres, principalmente entre } 1996 \\
\text { e } 2006 \text {, quando as diferenças entre as famílias } \\
\text { pobres e ricas que possuíam crianças menores } \\
\text { de cinco anos também diminuíram em termos } \\
\text { de poder aquisitivo; escolaridade da mãe; as- } \\
\text { sistência médica e serviços de água e saneamen- } \\
\text { to; e indicadores de saúde reprodutiva). }\end{array}$ \\
\hline Matijasevich et al. ${ }^{17}$ & Pelotas (RS) & $\begin{array}{l}\text { Um ano de idade: } 8,5-6,0 \\
\text { Dois anos de idade: } 13,9-5,0 \\
\text { Quatro anos de idade: } 10,9-3,6\end{array}$ & $\begin{array}{l}\text { Desigualdade socioeconômica (declínio tanto } \\
\text { entre os mais pobres quanto entre os mais ri- } \\
\text { cos, sendo significativo somente entre os mais } \\
\text { pobres e a um ano de idade). }\end{array}$ \\
\hline Ferreira et al. ${ }^{18}$ & Alagoas (SE) & $22,5-11,4$ & $\begin{array}{l}\text { Local de residência, acesso à água encanada, } \\
\text { número de pessoas no domicílio, número de } \\
\text { filhos, peso ao nascer, acesso ao pré-natal. }\end{array}$ \\
\hline
\end{tabular}

Nota: *Refere-se aos artigos: 1) Figueiroa JN, Alves JGB, Lira PIC, Batista Filho M. Evolução intergeracional da estatura no Estado de Pernambuco, Brasil, entre 1945 e 2006. 2 - aspectos analíticos. Cad Saúde Pública. 2012; 28(8):1468-78; e 2) Figueiroa JN, Alves JGB, Lira PIC, Batista Filho M. Evolução intergeracional da estatura no Estado de Pernambuco, Brasil, entre 1945 e 2006.1 - aspectos descritivos. Cad Saúde Pública. 2012; 28(7):1285-96.

\section{Reflexões sobre crescimento linear e equidade social}

A equidade em saúde implica, idealmente, que todos os indivíduos podem alcançar seu potencial de saúde. Nesse sentido, as iniquidades socioeconômicas representam diferenças sistemáticas socialmente produzidas e, portanto, modificáveis, que podem gerar desigualdade em termos de morbidade e mortalidade. Além do componente social, as iniquidades geográficas e culturais também se relacionam à equidade em saúde, sobretudo quando vinculadas à assistência. A condição própria de desenvolvimento e a dependência de outras pessoas para alcançar seu bem-estar de saúde, fazem das crianças indivíduos 
Quadro 3. Conclusões/considerações dos estudos sobre fatores associados ao declínio do deficit de estatura em crianças menores de cinco anos no Brasil.

\begin{tabular}{|c|c|c|}
\hline Fonte & Local de estudo & Conclusões/considerações \\
\hline Figueiroa et al. ${ }^{1 *}$ & Pernambuco & $\begin{array}{l}\text { Melhores condiçães socioeconômicas e ambientais favorecem o cres- } \\
\text { cimento estatural, tendências que sugerem a correção do deficit de } \\
\text { estatura no estado de Pernambuco; a manutenção do decréscimo } \\
\text { anual }(6,3 \%) \text { na proporção de crianças }<5 \text { anos de Pernambuco com } \\
\text { deficit de estatura levaria à ocorrência verificada no padrão interna- } \\
\text { cional de normalidade antropométrica da Organização Mundial da } \\
\text { Saúde }(2,3 \%) \text { em } 6-7 \text { anos, corrigindo, assim, o histórico retardo do } \\
\text { crescimento dessa população. }\end{array}$ \\
\hline Monteiro et al. ${ }^{3}$ & Brasil & $\begin{array}{l}\text { Contribuição positiva do desenvolvimento socioeconômico e das po- } \\
\text { líticas públicas favoráveis à equidade nas condições de vida e na des- } \\
\text { nutrição infantil, bem como na brecha do estado nutricional de crian- } \\
\text { ças nos quintil socioeconômicos superior e inferior. }\end{array}$ \\
\hline de Onis et al. ${ }^{4}$ & $\begin{array}{l}\text { Países em desenvolvimento, entre os } \\
\text { quais o Brasil }\end{array}$ & $\begin{array}{l}\text { Diminuição do retardo de crescimento nos países em desenvolvimen- } \\
\text { to e na América do Sul, bem como progressos desiguais nas diferen- } \\
\text { tes regiões do mundo. }\end{array}$ \\
\hline Lima et al. ${ }^{13}$ & Nordeste & $\begin{array}{l}\text { Tendências que sugerem o controle do deficit de estatura em menos } \\
\text { de dez anos na região Nordeste, se mantidas as melhorias relaciona- } \\
\text { das à escolaridade da mãe, saneamento, assistência à saúde e ante- } \\
\text { cedentes reprodutivos e, sobretudo, com o aumento do poder aqui- } \\
\text { sitivo familiar. }\end{array}$ \\
\hline Monteiro et al. ${ }^{14}$ & Brasil & $\begin{array}{l}\text { Tendências que sugerem o controle da desnutrição no Brasil como } \\
\text { problema de saúde pública em aproximadamente dez anos, se } \\
\text { mantidas as ações que têm favorecido o aumento do poder aquisiti- } \\
\text { vo dos mais pobres e o acesso a serviços de saúde, saneamento e } \\
\text { educação. }\end{array}$ \\
\hline $\begin{array}{l}\text { Monteiro \& Conde } \\
\text { et al. }{ }^{15}\end{array}$ & São Paulo & $\begin{array}{l}\text { Necessidade de políticas públicas que promovam o crescimento eco- } \\
\text { nômico, melhor distribuição da renda e universalização do acesso à } \\
\text { educação e a cuidados de saúde para a erradicação da desnutrição } \\
\text { infantil na cidade de São Paulo. }\end{array}$ \\
\hline Monteiro et al. ${ }^{16}$ & Brasil & $\begin{array}{l}\text { O desenvolvimento socioeconômico, aliado a políticas públicas visan- } \\
\text { do à igualdade no Brasil, têm sido acompanhados de declínio subs- } \\
\text { tancial da desnutrição infantil e redução da diferença de estado } \\
\text { nutricional entre crianças nos quintil socioeconômicos mais altos e } \\
\text { mais baixos. }\end{array}$ \\
\hline Matijasevich et al. ${ }^{17}$ & Pelotas (RS) & $\begin{array}{l}\text { Declínio das desigualdades socioeconômicas relacionadas ao deficit } \\
\text { de estatura. }\end{array}$ \\
\hline Ferreira et al. ${ }^{18}$ & Alagoas (SE) & $\begin{array}{l}\text { Transição nutricional, com redução na prevalência de deficit estatural } \\
\text { e incremento na frequência de obesidade, mediado por variáveis } \\
\text { socioeconômicas, demográficas e da situação de saúde. }\end{array}$ \\
\hline
\end{tabular}

Nota: *Refere-se aos artigos: 1) Figueiroa JN, Alves JGB, Lira PIC, Batista Filho M. Evolução intergeracional da estatura no Estado de Pernambuco, Brasil, entre 1945 e 2006. 2 - aspectos analíticos. Cad Saúde Pública. 2012; 28(8):1468-78; e 2) Figueiroa JN, Alves JGB, Lira PIC, Batista Filho M. Evolução intergeracional da estatura no Estado de Pernambuco, Brasil, entre 1945 e 2006.1 - aspectos descritivos. Cad Saúde Pública. 2012; 28(7):1285-96.

particularmente suscetíveis às iniquidades, principalmente socioeconômicas ${ }^{10}$. Nessa conjuntura, a baixa estatura é valorizada como indicador de mudanças relacionadas aos determinantes sociais de saúde e desenvolvimento, além de considerada a condição mais propícia na análise de metas de políticas públicas ${ }^{19}$.

O modelo de determinação do retardo do crescimento linear caracteriza-se por ser multicausal e complexo. Alimentação deficiente e/ou 
infecções recorrentes constituem as causas imediatas do deficit de estatura, inseridas em uma ampla gama de condições socioeconômicas relacionadas a um conjunto de circunstâncias materiais, ambientais e biológicas, as quais formam uma estrutura hierárquica, dinâmica e heterogênea que se reproduz entre gerações ${ }^{5,9,10}$. Além disso, a problemática deve compreender a influência das características dos modos da vida contemporânea, como o comportamento alimentar e a necessidade de cuidados terceirizados ${ }^{20-22}$. Nessa casuística, as transformações econômicas, sociais e demográficas acontecidas nas últimas décadas no Brasil têm sido destacadas por sua influência nas mudanças no perfil nutricional das crianças ${ }^{23}$, marcadamente a diminuição expressiva da prevalência de deficit de estatura ocorrida na região Nordeste ${ }^{24}$.

No entanto, esses avanços devem ser analisados com cautela, pois, por trás dos dados nacionais escondem-se diferenças importantes que marcam a persistência das iniquidades sociais relacionadas ao deficit de estatura no País. Nesse sentido, uma maior vulnerabilidade manifesta-se na região Norte ${ }^{24}$ e em grupos populacionais específicos, como os indígenas ${ }^{25}$ e os beneficiários do Programa Bolsa Família ${ }^{26}$, com prevalências respectivas de deficit de estatura de 14,8, 26,0 e $12,2 \%$ nas crianças menores de cinco anos. Também é necessário destacar outros grupos marginalizados que, apesar de inseridos em ambientes de exclusão social, têm sido pouco considerados nas pesquisas. Nesse sentido, podem ser citados, por exemplo, estudos desenvolvidos com crianças quilombolas ${ }^{27}$, residentes em favelas ${ }^{28}$, de famílias de trabalhadores rurais sem terra ${ }^{29}$ e ribeirinhas ${ }^{30}$, os quais apontaram, respectivamente, taxas de deficit no crescimento linear de 31,1, 8,6, 8,0 e $17,3 \%$.

Assim, o debate sobre o crescimento linear das crianças brasileiras deve expandir para o contexto da segurança alimentar e nutricional, parâmetro estabelecido como importante indicador de iniquidade social ${ }^{31}$. Nesse sentido, indica-se, apesar das discrepâncias entre as prevalências de insegurança alimentar e de deficit de estatura constatadas na literatura ${ }^{32,33}$, associação entre esses dois indicadores ${ }^{34}$, com prejuízos no potencial de crescimento linear nas crianças que sofrem com algum nível de insegurança alimentar, segundo dados nacionais ${ }^{35}$. Essa conjuntura implica o entendimento da baixa estatura como evento, ao mesmo tempo, próximo, distinto e derivado da insegurança alimentar ${ }^{32,33}$. Ressalta-se ainda que, possivelmente, exista um novo momento no qual o deficit de estatura e a insegurança alimentar estejam dissociados das deficiências de macronutrientes, sendo substituídos pela influência das carências de micronutrientes ${ }^{32}$, com destaque para aquelas de maior repercussão no crescimento linear (anemia, hipovitaminose A e deficiência de zinco) $)^{36}$.

Por outro lado, é fundamental advertir a coexistência da desnutrição crônica com o excesso de peso como uma das caraterísticas marcantes do processo de transição nutricional brasileiro ${ }^{37}$. Esse cenário tipifica as transformações econômicas, sociais e demográficas, o processo de modernização, e a insegurança alimentar enquanto propulsores de mudanças, não apenas em relação à estatura das crianças, senão, também, em relação ao contexto da obesidade e das doenças associadas à má alimentação ${ }^{37-39}$.

No contexto deste estudo, reforça-se, com respaldo na literatura já publicada, a determinação social da baixa estatura pela influência da renda familiar, da escolaridade materna, das condições de saneamento básico e dos serviços básicos de saúde. A renda familiar é reconhecida como o principal fator relacionado à melhoria no acesso à alimentação ${ }^{40}$. A escolaridade materna relaciona-se diretamente com o cuidado à criança e com a eficiência na utilização dos recursos financeiros e dos serviços de saúde ${ }^{41}$. O saneamento básico, apesar das grandes lacunas de cobertura que ainda persistem ${ }^{42}$, tem forte relação com a proteção contra doenças infecto-parasitárias, as quais expõem as crianças a um processo de agravamento do estado nutriciona| ${ }^{41,43}$. Em relação aos serviços básicos de saúde, a Atenção Primária des- 
taca-se na garantia do direito à saúde, trazendo ações, como o acompanhamento do crescimento e do desenvolvimento das crianças, a suplementação com micronutrientes e a promoção do aleitamento materno, as quais repercutem positivamente no estado nutricional e na situação de saúde ${ }^{36,43,44}$.

Nessa conjuntura, o governo federal tem priorizado programas sociais com ações intersetoriais destinadas a mitigar a pobreza e a miséria ${ }^{45}$, como o Plano Brasil sem Miséria ${ }^{46}$, o Programa Bolsa Família ${ }^{26}$ e o Programa Saúde na Escola ${ }^{47}$. No contexto do setor saúde, mais especificamente da Atenção Primária à Saúde, as ações de alimentação e nutrição apresentam-se, também, com especificação intersetorial, envolvendo os setores saúde, educação, assistência social e trabalho, além do componente saúde do Programa Bolsa Família ${ }^{48}$. Ainda de modo a melhorar o acesso a alimentos saudáveis pela população, principalmente a de baixa renda, a implementação das políticas de segurança alimentar e nutricional considera a necessidade da articulação dos setores de produção, comercialização e consumo de alimentos ${ }^{49}$.

Assim, cabe ressaltar que a combinação de fatores múltiplos como o crescimento econômico, a estabilidade monetária, o aumento do salário mínimo, a universalização da educação e da saúde e a expansão do Programa Bolsa Família tem contribuído de maneira importante para a obtenção de níveis satisfatórios dos indicadores sociais e de saúde, traduzindo-se em benefícios nutricionais. É importante destacar, portanto, que políticas sociais centradas na garantia da equidade entre as famílias de diferentes regiões, áreas e grupos socioculturais do País, com enfoque no aumento do poder aquisitivo e do acesso aos serviços essenciais de educação, saneamento e saúde dos mais vulneráveis, devem ser priorizadas como forma de propiciar maior impacto nos problemas de desnutrição crônica persistentes no Brasil.

Esse tema, no entanto, não se esgota aqui. Apesar de questões a esse respeito terem sido levantadas, a discussão epistemológica faz-se necessária no sentido de promover rigor científico com base nos critérios de cientificidade e de verdade, profunda e responsavelmente repensados, como levantado por outros autores em temas similare ${ }^{50}$. Nesse sentido, há que ressaltar a imperiosa necessidade de advogar contra o reducionismo científico na área da saúde, incentivando, por outro lado, pesquisas com análises e atuações abrangentes e interconectadas, antagônicas às propostas unidirecionais e unívocas ${ }^{51}$.

\section{O N C L U S Ã O}

O presente artigo analisa a redução da baixa estatura entre as crianças brasileiras menores de cinco anos, contextualizando o tema na perspectiva da equidade social. Apesar do importante declínio no deficit estatural ao longo dos anos e do progresso nas condições de vida da população, prevalências discrepantes, intra e inter-regionais, ainda são observadas, com vulnerabilidade marcada em grupos socioculturais desfavorecidos. O enfrentamento das disparidades existentes deve estar baseado na manutenção e no reforço das políticas e programas públicos, com ações intersetoriais, direcionados aos grupos desprivilegiados. Também faz-se imprescindível o adequado monitoramento do estado nutricional das crianças com a finalidade de atender às necessidades dos grupos vulneráveis precocemente, bem como direcionar as política públicas.

Como a nutrição adequada de segmentos marginalizados continua sendo um grande desafio para as políticas públicas no Brasil, sugere-se que as pesquisas sejam direcionadas a essas camadas da população. As análises, nesse sentido, devem abordar, além da própria questão antropométrica, o contexto na perspectiva da segurança alimentar, das deficiências de micronutrientes, das implicações dos modos da vida contemporânea e da coexistência com o sobrepeso/obesidade.

\section{REFERÊ NCIAS}

1. Figueiroa JN, Alves JGB, Lira PIC, Batista Filho M. Evolução intergeracional da estatura no Estado de 
Pernambuco, Brasil, entre 1945 e 2006. 2 - aspectos analíticos. Cad Saúde Pública. 2012; 28(8):1468-78.

2. Leal VL, Lira PIC, Menezes RCI, Oliveira JS, Sequeira SLA, Andrade SLS, et al. Fatores associados ao declínio do déficit estatural em crianças e adolescentes em Pernambuco. Rev Saúde Pública. 2012; 46(2):234-41.

3. Monteiro CA, Benicio MH, Conde $\mathrm{WL}$, Konno S, Lovadino AL, Barros AJD, et al. Narrowing socioeconomic inequality in child stunting: The Brazilian experience, 1974-2007. Bull World Health Organ. 2010; 88:305-11.

4. de Onis M, Frongillo EA, Blössner M. Is malnutrition declining? An analysis of changes in levels of child malnutrition since 1980. Bull World Health Organ. 2000; 78(10):1222-33.

5. de Onis M, Blössner M, Borghi E. Prevalence and trends of stunting among pre-school children, 1990-2020. Public Health Nutr. 2012; 15(1):142-8.

6. United Nations. The Millennium Development Goals Report. New York: United Nations; 2007.

7. Ananias P. Accelerating the reduction of maternal and child malnutrition: Contributions to the debate based on the Brazilian experience. SCN News. 2008; 36:8-11.

8. Neri M. Miséria, desigualdade e políticas de renda: o real do Lula. Rio de Janeiro: Fundação Getúlio Vargas; 2007.

9. Sánchez-Abanto J. Evolución de la desnutrición crónica en menores de cinco años en el Perú. Rev Peru Med Exp Salud Pública. 2012; 29(3):402-5.

10. Barros FC, Victora CG, Scherpbier R, Gwatkin D. Socioeconomic inequities in the health and nutrition of children in low/middle income countries. Rev Saúde Pública. 2010; 44(1):1-16.

11. Van de Poel E, Hosseinpoor AR, Speybroeck N, Van Ourti T, Vega J. Socioeconomic inequality in malnutrition in developing countries. Bull World Health Organ. 2008; 86(6):282-91.

12. World Health Organization. Length/height-for-age, weight-for-age, weight-for-length, weight-for-height and body mass index-for-age. Geneva: WHO; 2006.

13. Lima AL, Silva AC, Konno SC, Conde WL, Benicio $\mathrm{MH}$, Monteiro CA. Causes of the accelerated decline in child undernutrition in Northeastern Brazil (1986-1996-2006). Rev Saúde Pública. 2010; 44(1):17-27.

14. Monteiro CA, Benicio MH, Konno SC, Silva ACF, Lima AL, Conde WL. Causes for the decline in child under-nutrition in Brazil, 1996-2007. Rev Saúde Pública. 2009; 43(1):35-43.

15. Monteiro CA, Conde WL. Tendência secular da desnutrição e da obesidade na infância na cidade de
São Paulo (1974-1996). Rev Saúde Pública. 2000; 34(Supl. 6):52-61.

16. Monteiro CA, Benicio MHDA, Conde WL, Konno SC, Lima ALL, Barros AJD, et al. Desigualdades socioeconômicas na baixa estatura infantil: a experiência brasileira, 1974-2007. Estud Av. 2013; 27(78):36-49.

17. Matijasevich A, Santos IS, Menezes AM, Barros AJ, Gigante DP, Horta BL, et al. Trends in socioeconomic inequalities in anthropometric status in a population undergoing the nutritional transition: Data from 1982, 1993 and 2004 Pelotas birth cohort studies. BMC Public Health. 2012; 12:511.

18. Ferreira HS, Cesar JÁ, Assunção ML, Horta BL. Time trends (1992-2005) in undernutrition and obesity among children under five years of age in Alagoas State, Brazil. Cad Saúde Pública. 2013; 29(4):793-800.

19. Bacallao J, Peña M, Díaz A. Desnutrición crónica en las bases biosociales para la salud y el desarrollo. Rev Panam Salud Pública. 2012; 32(2):145-50.

20. Brasil. Ministério da Saúde. Marco de referência da educação alimentar e nutricional para as políticas públicas. Brasília: Ministério da Saúde; 2011.

21. Goulart RMM, Banduk MLS, Taddei JAAC. Uma revisão das ações de nutrição e do papel do nutricionista em creches. Rev Nutr. 2010; 23(4):655-65. http://dx.doi.org/10.1590/S1415-527320100004 00015

22. Maranhão DG, Sarti CA. Creche e família: uma parceria necessária. Cad Pesq. 2008; 38(133):171-94.

23. Menezes RCE, Lira PIC, Leal VS, Oliveira JS, Santana SCS, Sequeira LAS, et al. Determinantes do déficit estatural em menores de cinco anos no Estado de Pernambuco. Rev Saúde Pública. 2011; 45(6):1079-87.

24. Monteiro CA, Conde WL, Konno SC, Lima AL, Silva AC, Benicio MH. Avaliação antropométrica do estado nutricional de mulheres em idade fértil e crianças menores de cinco anos. In: Brasil, Ministério da Saúde. Pesquisa Nacional de Demografia e Saúde da Criança e da Mulher: PNDS 2006: dimensões do processo reprodutivo e da saúde da criança. Brasília: Ministério da Saúde; 2009. p.213-30.

25. Horta BL, Santos RV, Welch JR, Cardoso AM, Santos $\mathrm{J}$, Assis AMO, et al. Nutritional status of indigenous children: Findings from the First National Survey of Indigenous People's Health and Nutrition in Brazil. Int J Equity Health. 2013; 12:23.

26. Brasil. Ministério do Desenvolvimento Social e Combate à Fome. Avaliação da evolução temporal do estado nutricional das crianças de 0 a 5 anos beneficiárias do Programa Bolsa Família (PBF), acompanhadas nas condicionalidades de saúde. 
Brasília: Ministério do Desenvolvimento Social e Combate à Fome; 2014.

27. Guimarães RCR, Silva HP. Estado nutricional e crescimento de crianças quilombolas de diferentes comunidades do estado do Pará. Amazônica. 2015; 7(1):186-209.

28. Silveira KBR, Alves JFR, Ferreira HS, Sawaya AL, Florêncio TMMT. Association between malnutrition in children living in favelas, maternal nutritional status, and environmental factors. J Pediatr. 2010; 86(3):215-20.

29. Lang RMF, Almeida CCB, Taddei JA. Segurança alimentar e nutricional de crianças menores de dois anos de famílias de trabalhadores rurais Sem Terra. Ciênc Saúde Colet. 2011; 16(7):3111-8.

30. Silva SA, Moura EC. Determinantes do estado de saúde de crianças ribeirinhas menores de dois anos de idade do Estado do Pará, Brasil: um estudo transversal. Cad Saúde Pública. 2010; 26(2):273-85.

31. Bezerra TA, Figueroa Pedraza D. (In)segurança alimentar entre famílias com crianças menores de cinco anos residentes em área de vulnerabilidade social de Campina Grande, Paraíba. Rev Nutr. 2015; 18(6):655-65.

32. Figueroa Pedraza D, Queiroz D, Paiva AA, Cunha MAL, Lima ZN. Seguridad alimentaria, crecimiento y niveles de vitamina $A$, hemoglobina y zinc en niños preescolares del Nordeste de Brasil. Ciênc Saúde Colet. 2014; 19(2):641-50.

33. Oliveira JS, Lira PIC, Andrade SLLS, Sales AC, Maia SR, Batista Filho M. Insegurança Alimentar e estado nutricional de crianças de São João do Tigre, no semi-árido do Nordeste. Rev Bras Epidemiol. 2009; 12(3):413-23.

34. Morais DC, Dutra LV, Franceschini SCC, Priore SE. Insegurança alimentar e indicadores antropométricos, dietéticos e sociais em estudos brasileiros: uma revisão sistemática. Ciênc Saúde Colet. 2014; 19(5):1475-88.

35. Santos LPD, Gigante DP. Relação entre insegurança alimentar e estado nutricional de crianças brasileiras menores de cinco anos. Rev Bras Epidemiol. 2013; 16(4):984-94.

36. Figueroa Pedraza D. Evidências do impacto da suplementação múltipla com micronutrientes no crescimento de pré-escolares. Rev Bras Saúde Matern Infant. 2014; 14(1):17-37.

37. Conde WL, Monteiro CA. Nutrition transition and double burden of undernutrition and excess of weight in Brazil. Am J Clin Nutr. 2014; 100(Supl.):1617S-22.

38. Wanderley EM, Ferreira VA. Obesidade: uma perspectiva plural. Ciênc Saúde Colet. 2010; 15(1):185-94.

39. Souza MM, Pedraza DF, Menezes TN. Estado nutricional de crianças assistidas em creches e situação de (in)segurança alimentar de suas famílias. Ciênc Saúde Colet. 2012; 17(12):3425-36.
40. Facchini LA, Nunes BP, Motta JVS, Tomasi E, Silva SM, Thumé $E$, et al. Insegurança alimentar no Nordeste e Sul do Brasil: magnitude, fatores associados e padrões de renda per capita para redução das iniquidades. Cad Saúde Pública. 2014; 30(1):161-74.

41. Rissin A, Figueiroa JN, Benício MHD, Batista Filho M. Retardo estatural em menores de cinco anos: um estudo "baseline". Ciênc Saúde Colet. 2011; 16(10):4067-76.

42. Borja PC. Política pública de saneamento básico: uma análise da recente experiência brasileira. Saúde Soc. 2014; 23(2):432-47.

43. Imdad A, Sadig K, Bhutta ZA. Evidence-based prevention of childhood malnutrition. Curr Opin Clin Nutr Metab Care. 2011; 14(3):276-85.

44. Ashworth A, Shrimpton R, Jamil K. Growth monitoring and promotion: Review of evidence of impact. Maternal Child Nut. 2008; 4:86-117.

45. Anschau FR, Matshuo T, Segall-Corrêa AM. Insegurança alimentar entre beneficiários de programas de transferência de renda. Rev Nutr. 2012; 25(2):177-89. http://dx.doi.org/10.1590/S1415-52 732012000200001

46. Brasil. Presidência da República. Decreto n 7.492, de 2 de junho de 2011. Institui o Plano Brasil sem Miséria. Diário Oficial da União. 2011; 3 jun.

47. Ferreira IRC, Moysés SJ, França BHS, Carvalho ML, Moysés ST. Percepções de gestores locais sobre a intersetorialidade no Programa Saúde na Escola. Rev Bras Educ. 2014; 19(56):61-76.

48. Jaime PC, Silva ACF, Lima AMC, Bortolini GA. Ações de alimentação e nutrição na atenção básica: a experiência de organização no Governo Brasileiro. Rev Nutr. 2011; 24(6):809-24. http://dx.doi.org/ 10.1590/S1415-52732011000600002

49. Burlandy L. A construção da política de segurança alimentar e nutricional no Brasil: estratégias e desafios para a promoção da intersetorialidade no âmbito federal de governo. Ciênc Saúde Colet. 2009; 14(3):851-60.

50. Minayo MCS. A busca da verdade no campo científico da saúde. Ciênc Saúde Colet. 2012; 18(10):2806-12.

51. Amparo-Santos L. Avanços e desdobramentos do marco de referência da educação alimentar e nutricional para políticas públicas no âmbito da universidade e para os aspectos culturais da alimentação. Rev Nutr. 2013; 26(5):595-600. http://dx.doi.org/ 10.1590/S1415-52732013000500010

Recebido: maio 5, 2015

Versão final: julho 17, 2015

Aprovado: setembro 17, 2015 